\title{
COUNTABLE PRODUCTS OF SCATTERED PARACOMPACT SPACES
}

\author{
MARY ELLEN RUDIN AND STEVE WATSON
}

\begin{abstract}
ABSTRACr. In this paper we prove that the product of countably many scattered paracompact spaces is even ultraparacompact.
\end{abstract}

Telgársky [1] has shown that scattered paracompact spaces are ultraparacompact. Verbally, H. Martin has asked if a product of countably many spaces with exactly one nonisolated point has to be paracompact. We prove

THEOREM. The product of countably many scattered paracompact spaces is ultraparacompact.

All spaces are assumed Hausdorff. A space is ultraparacompact if every open cover has a disjoint open refinement. We occasionally use the word refinement when less than the whole space is covered: if so the covered subspace is always mentioned. A scattered space $X$ is $\cup_{\alpha<\lambda} X^{\alpha}$ for some minimal ordinal $\lambda$ where, for $\alpha<\lambda, X^{\alpha}$ is the set of all isolated points of $X-\cup_{\beta<\alpha} X^{\beta}$. The order of $X$ is $\lambda$ and rank of $x \in X$ is the $\alpha<\lambda$ with $x \in X^{\alpha}$. We say a subset $A$ of $X$ is topped if $A$ has a unique point of maximal rank (i.e. the top of $A$ ). For completeness we prove

LEMMA. Suppose $\mathcal{G}$ is an open cover of a paracompact scattered space $Y$. Then $\mathcal{G}$ has a disjoint, topped, open refinement (covering $Y$ ).

Proof. Suppose (order $Y$ ) is minimal for the lemma to fail.

Case (1). (Order $Y$ ) is a limit. There is a locally finite open refinement $\mathcal{H}$ of $\mathcal{G}$ by sets whose closures have order less than (order $Y$ ). Let $\mathscr{K}$ be a locally finite closed refinement of $\mathcal{H}$.

For $H \in \mathcal{H}$, let $K_{H}=\cup\{K \subset H \mid K \in \mathscr{K}\}$. Since (order $\bar{H}$ ) $<$ (order $Y$ ), there is a disjoint, open in $\bar{H}$, refinement $\mathcal{g}_{H}$ of $\left\{H, \bar{H}-K_{H}\right\}$ covering $\bar{H}$. Let $g_{H}=\left\{J \in \mathcal{G}_{H} \mid\right.$ $\left.J \cap K_{H} \neq \varnothing\right\}$.

Since $G=\cup_{H \in \mathcal{X}} \mathscr{G}_{H}$ is a locally finite cover of $Y$ by clopen sets, by the standard technique of subtraction one can find an open, disjoint refinement $\mathcal{L}$ of $\mathscr{G}$ covering $Y$. Since (order $L)<\left(\right.$ order $Y$ ), each $L \in \mathcal{L}$ can be covered by a set $\delta_{L}$ of disjoint, topped open sets. Thus $\cup_{L \in \mathcal{E}} \delta_{L}$ is a disjoint, topped open refinement of $\mathcal{G}$ as desired.

Case (2). (Order $Y)=\alpha+1$. Let $Y^{\alpha}$ be the set of all points of $Y$ of rank $\alpha$. Since $Y^{\alpha}$ is a closed discrete subset of the paracompact $Y$, there is a disjoint open

Received by the editors December 28, 1982 and, in revised form, February 16, 1983.

1980 Mathematics Subject Classification. Primary 54B10, 54D18. 
refinement $\mathcal{H}$ of $\mathcal{G}$ covering $Y^{\alpha}$ with each member of $\mathcal{H}$ containing precisely one point of $Y^{\alpha}$. Choose an open set $U$ with $Y^{\alpha} \subset U \subset \bar{U} \subset \cup \mathcal{H}$. Since (order $(Y-U))<(\operatorname{order} Y)$, there is a disjoint, topped, open in $Y-U$, refinement $\mathcal{K}$ of $\{\cup \mathcal{H}-U\} \cup\{G-\bar{U} \mid G \in \mathcal{G}\}$ covering $Y-U$. Taking $\mathscr{g}=\{K \in \mathscr{K} \mid K \cap \bar{U}=$ $\varnothing\}, \mathscr{G} \cup\{H-\cup \mathscr{F} \mid H \in \mathcal{H}\}$ is a disjoint, topped, open refinement of $\mathcal{G}$ covering $Y$ as desired.

The lemma is proved.

Proof of THE THEOREM. Suppose that for each $n \in \omega, X_{n}$ is a paracompact scattered space, $X=\prod_{n \in \omega} X_{n}$, and $\Theta$ is an open cover of $X$.

Let $\Omega$ be the set of all subsets of $X$ which cannot be covered by any disjoint, open refinement (not necessarily covering $X$ ) of $\theta$. We make frequent use of: (*) If a member of $\Omega$ is the union of disjoint clopen sets, then one of these sets is in $\Omega$.

We assume $X \in \Omega$ in order to get a contradiction.

For each $i \in \omega$ we presently choose $k_{i} \in \omega$ and a function $f_{i}$ having domain $\omega$ such that $f_{i}(n)$ is a topped clopen subset of $X_{n}$ if $n<k_{i}, f_{i}(n)=X_{n}$ if $n \geqslant k_{i}$, and $\Pi_{n \in \omega} f_{i}(n) \in \Omega$.

Let $k_{0}=0$; thus each $f_{0}(n)=X_{n}$ and $\Pi_{n \in \omega} f_{0}(n)=X \in \Omega$.

Having defined $k_{i}$ and $f_{i}$ we consider two cases.

Case (1). For each $n<k_{i}$, there is a clopen $U_{n}$ in $X_{n}$ with (top $\left.f_{i}(n)\right) \in U_{n}$ and $\left(\prod_{n<k_{i}} U_{n} \times \Pi_{n \geqslant k_{i}} X_{n}\right) \notin \Omega$.

By $(*)$, there is $m<k_{i}$ with at least $\left(f_{i}(m)-U_{m}\right) \times \Pi_{n \neq m} f(n) \in \Omega$. By the Lemma, there is a disjoint, topped, open cover $\mathscr{V}$ of $f_{i}(m)-U_{m}$. Define $k_{i+1}=$ $k_{i}, f_{i+1}(n)=f_{i}(n)$ for $n \neq m$, and choose $f_{i}(m) \in \mathcal{V}$, by $(*)$, so that $\prod_{n \in \omega} f_{i+1}(n) \in$ $\Omega$.

Case (2). Not Case (1). By the Lemma, there is a disjoint, topped, open cover $\mathscr{U}$ of $X_{k_{i}}$. Define $k_{i+1}=k_{i}+1, f_{i+1}(n)=f_{i}(n)$ for $n \neq k_{i}$, and choose $f_{i+1}\left(k_{i}\right) \in \mathcal{Q}$, by (*), so that $\prod_{n \in \omega} f_{i+1}(n) \in \Omega$.

Since Case (1) implies $k_{i+1}=k_{i}$ and $\operatorname{rank}\left(\operatorname{top} f_{i+1}(m)\right)<\operatorname{rank}\left(\operatorname{top} f_{i}(m)\right)$ for some $m<k_{i}$, and there is no infinite decreasing sequence of ordinals, Case (2) must hold for infinitely many $i \in \omega$.

Since Case (2) implies $k_{i+1}>k_{i}$, for every $n \in \omega$, there is $i_{n} \in \omega$ with $n<k_{i_{n}}$. Hence $f_{i_{n}}(n)$ has a top. Since $\operatorname{rank}\left(\operatorname{top} f_{i+1}(n)\right) \leqslant \operatorname{rank}\left(\operatorname{top} f_{i}(n)\right)$ we can choose $i_{n}$ sufficiently large so that, for all $i \geqslant i_{n}, \operatorname{rank}\left(\operatorname{top} f_{i}(n)\right)=\operatorname{rank}\left(\operatorname{top} f_{i_{n}}(n)\right)$. Thus, for $i \geqslant i_{n}, \operatorname{top}\left(f_{i}(n)\right)=\operatorname{top}\left(f_{i_{n}}(n)\right)$.

If $t$ is the point of $X$ with $t(n)=\operatorname{top}\left(f_{i_{n}}(n)\right)$, then $t \in O \in O$. So there is $k \in \omega$ and for each $n<k$ a clopen $O_{n}$ in $X_{n}$ such that $t(n) \in O_{n}$ and $\left(\prod_{n<k} O_{n} \times \prod_{n \geqslant k} X_{n}\right)$ $\subset O$.

If $i \geqslant i_{n}$ for all $n<k, n<k$ implies $n<k_{i}$ and top $\left(f_{i}(n)\right)=t(n)$. So regardless of $\operatorname{top}\left(f_{i}(n)\right)$ for $k \leqslant n<k_{i}$, Case (1) holds for $i$. This contradicts the fact that Case (2) holds infinitely often.

\section{REFERENCES}

1. R. Telgársky, Total paracompactness and paracompact dispersed spaces, Bull. Acad. Polon. Sci. Sér. Sci. Math. Astronom. Phys. 16 (1968), 567-572.

Department of Mathematics, University of Wisconsin, Madison, Wisconsin 53706 